\title{
Quenched-annealed density functional theory for interfacial behavior of hard rods at a hard rod matrix
}

\author{
David L. Cheung ${ }^{1, a)}$ and Matthias Schmidt ${ }^{2}$ \\ ${ }^{1}$ Department of Chemistry and Centre for Scientific Computing, University of Warwick, Coventry CV4 7AL, \\ United Kingdom \\ ${ }^{2}$ Theoretische Physik II, Universität Bayreuth, D-95440 Bayreuth, Germany and H.H. Wills Physics \\ Laboratory, University of Bristol, Tyndall Avenue, Bristol BS8 1TL, United Kingdom
}

(Received 17 September 2009; accepted 3 November 2009; published online 4 December 2009)

\begin{abstract}
We consider a system of mobile hard rods that are immersed in an isotropic matrix of hard rods with quenched positions and orientations. Using quenched-annealed density functional theory the disorder-averaged excess free energy functional is approximated by an Onsager second virial form, which is valid in the limit of large length-to-thickness aspect ratio of the particles. We find that inside the bulk isotropic matrices the isotropic-nematic phase transition occurs at higher values of the chemical potential than in the pure system, shifted proportionally to the product of the matrix density and the matrix-fluid excluded volume. We investigate adsorption and penetration behavior of the annealed rods at the planar surface of a porous rod matrix, considering both perpendicular and parallel boundary conditions of the nematic director far from the surface. (C) 2009 American Institute of Physics. [doi:10.1063/1.3267728]
\end{abstract}

\section{INTRODUCTION}

The effect of quenched disorder on the isotropic-nematic phase transition is a topic of active research. ${ }^{1,2}$ While the Imry-Ma argument ${ }^{3}$ rules out the existence of true longranged order in a nematic phase that is exposed to the influence of quenched random orienting fields, it was shown that quasi-long-range order can exist in nematics confined in random porous media. ${ }^{4}$ In unconfined systems nematic ordering in lyotropic systems can be understood on a microscopic level via repulsive hard core interactions of nonspherical particles. Onsager's treatment of the isotropic-nematic transition of the hard rod fluid acts as a paradigm. ${ }^{5-7}$ In order to study orientationally ordered liquid crystalline phases, density functional theory ${ }^{8,9}$ (DFT) constitutes a powerful tool. Onsager theory can be viewed as a DFT in which the lowdensity expansion of the (exact) free energy functional is truncated at the second virial level. A simple microscopic approach to porous substances is to use immobilized (quenched) particle configurations of a model fluid to represent the porous material. An equilibrated (annealed) fluid forms the adsorbate. A treatment of such quenched-annealed (QA) mixtures can be based on the replica trick, as is done in liquid state integral equation theory for QA mixtures, ${ }^{10-12}$ also applied to models with orientational degrees of freedom, such as dipolar interactions. ${ }^{13-15}$

Extending earlier approaches, ${ }^{16,17}$ a DFT was formulated for QA mixtures. ${ }^{18,19}$ The variational principle $^{18}$ for the disorder-averaged grand potential was derived using the replica procedure, ${ }^{19}$ as well as from first principles. ${ }^{20}$ Applying the theory requires an approximation for the disorderaveraged free energy functional. Approaches such as meanfield theory ${ }^{21-23}$ and fundamental measure theory ${ }^{18}$ have

${ }^{a)}$ Electronic mail: david.cheung@warwick.ac.uk. been used successfully for simple model systems; see Ref. 24 for an overview. The QA DFT has also been used to study phenomena in more complex models, such as capillary condensation in pores with rough walls $\mathrm{s}^{25}$ and adsorption in slitlike pores modified with chain molecules ${ }^{26}$ on amorphous and microporous silica materials, ${ }^{21}$ in pillared slitlike pores, ${ }^{27}$ and on surfaces modified with brushlike chain structures. ${ }^{28}$

In simple rod models the QA DFT was used to investigate the structure of hard spheres immersed in random fiber networks, ${ }^{29}$ and the isotropic-nematic transition of (thin) annealed hard rods immersed in a matrix of quenched hard spheres. ${ }^{30}$ In the latter system it was found that upon increasing disorder (sphere density) nematic ordering persists at high rod densities for the system sizes accessible to Monte Carlo computer simulations. The true long-range behavior of the model could not be accessed though. In simulations finite-size effects are present and the DFT is essentially a mean-field treatment. Studying QA systems with simulations is challenging, as the average over the quenched disorder needs to be carried out explicitly by simulating many different realizations of the random matrix. ${ }^{31,32}$ The fact that this average is implicitly taken renders the QA DFT a computationally very efficient framework.

In this paper we consider a binary QA mixture of hard rods, both of large aspect ratio (of length and width). We use a QA version of the Onsager functional, which possesses a relatively simple mathematical structure and is expected to give reliable results in the case of large aspect ratio (thin rods), and investigate bulk and interfacial behavior at the surface of an isotropic matrix of quenched hard rods. We are inspired by the fact that the equilibrium analog of the model considered, namely, binary hard rod mixtures, displays rich equilibrium phenomenology of bulk and interfacial behavior. $^{33-37}$ 
The paper is organized as follows. In Sec. II we define the model and lay out the theory. Section III describes the computational methods used. Section IV presents results for interfacial behavior at a matrix of quenched rods. In Sec. V conclusions are drawn.

\section{THE MODEL, REPLICA TRICK, AND DENSITY FUNCTIONAL THEORY}

We consider an annealed hard rod fluid (species 1) immersed in a matrix of quenched hard rods (species 0 ). The particles of both species $i=0,1$ have ellipsoidal shape and are characterized by the (rod) length $a_{i}$ and diameter $b_{i}$. The pair interaction potential between a rod of species $i$ at space point $\mathbf{r}$ and orientation $\mathbf{u}$ (along the rod axis) and a rod of species $j$ at space point $\mathbf{r}^{\prime}$ and orientation $\mathbf{u}^{\prime}$ is $\phi_{i j}(\mathbf{r}$ $\left.-\mathbf{r}^{\prime}, \mathbf{u}, \mathbf{u}^{\prime}\right)=\infty$ if both particles overlap and zero otherwise. The one-body distribution of species $i$ is denoted by $\rho_{i}(\mathbf{r}, \mathbf{u})$. Furthermore we consider a replicated system that contains $s+1$ species. Species $i=2, \ldots, s$ are replicas of species 1 , in the sense that particles of these species interact in the same way with species 0 as species 1 does, $\phi_{0 i}=\phi_{01}$, and that the intraspecies interactions are the same, $\phi_{i i}=\phi_{11}, i>0$. However, particles of different replicas are mutually noninteracting, hence $\phi_{i j}=0$ for $i \neq j>0$.

We first review the replica procedure for the bulk free energy. The grand canonical trace over the degrees of freedom of species 0 is written as

$$
\int d \mathbf{0} \equiv \sum_{N_{0}=0}^{\infty} \frac{\mathrm{e}^{\beta \mu_{0} N_{0}}}{\Lambda_{0}^{3 N_{0}}} \int d \mathbf{r}_{0}^{(1)} \int d \mathbf{u}_{0}^{(1)} \cdots \int d \mathbf{r}_{0}^{\left(N_{0}\right)} \int d \mathbf{u}_{0}^{\left(N_{0}\right)},
$$

where the lower index labels the species and the upper index labels the $N_{i}$ individual particles, $\mu_{i}$ is the chemical potential, $\Lambda_{i}$ is the thermal wavelength of species $i$, and $\beta=1 / k_{B} T$, where $k_{B}$ is Boltzmann's constant and $T$ is the absolute temperature. The integrals over $\mathbf{r}_{0}^{(1)}, \ldots, \mathbf{r}_{0}^{\left(N_{0}\right)}$ run over space and those over $\mathbf{u}_{0}^{(0)}, \ldots, \mathbf{u}_{0}^{\left(N_{0}\right)}$ run over the unit sphere. The trace for the annealed species, $\int d \mathbf{1}$, and that for the replicated components, $\int d \mathbf{2}, \ldots, \int d \mathbf{s}$, are defined accordingly, i.e., by replacing species index 0 by $1,2, \ldots, s$ in Eq. (1), respectively. The total potential energy from intraspecies interactions, $V_{i i}$, and from fluid-matrix interactions, $V_{0 i}$, for $i=0,1, \ldots, s$, are given by

$$
\begin{aligned}
V_{i i} & =\sum_{k=1}^{N_{i}} \sum_{l=k+1}^{N_{i}} \phi_{i i}\left(\mathbf{r}_{k}^{(i)}-\mathbf{r}_{l}^{(i)}, \mathbf{u}_{k}^{(i)}, \mathbf{u}_{l}^{(i)}\right), \\
V_{0 i} & =\sum_{k=1}^{N_{0}} \sum_{l=1}^{N_{i}} \phi_{0 i}\left(\mathbf{r}_{k}^{(0)}-\mathbf{r}_{l}^{(i)}, \mathbf{u}_{k}^{(0)}, \mathbf{u}_{l}^{(i)}\right) .
\end{aligned}
$$

The QA Helmholtz free energy $F_{1}$ of the adsorbate in the presence of the matrix, averaged over all matrix realizations, is defined via

$$
-\beta F_{1}=Z_{0}^{-1} \int d \mathbf{0} \mathrm{e}^{-\beta V_{00}} \ln \int d \mathbf{1} \mathrm{e}^{-\beta\left(V_{01}+V_{11}\right)},
$$

where the grand partition sum for the quenched species is

$$
Z_{0}=\int d \mathbf{0} \mathrm{e}^{-\beta V_{00}}
$$

An alternative formulation of the right hand side of Eq. (4) can be based on the replica procedure: One considers a replicated system, whose (equilibrium) grand partition sum is

$$
Z=\int d \mathbf{0} \int d \mathbf{1} \cdots \int d \mathbf{s e}^{-\beta\left(V_{00}+\Sigma_{i=1}^{s} V_{0 i}+\Sigma_{i=1}^{s} V_{i i}\right)} .
$$

By rearranging the orders of integration in Eq. (6) and exploiting the symmetry of the replicated pair interactions this can be written as

$$
Z=\int d \mathbf{0} \mathrm{e}^{-\beta V_{00}}\left[\int d \mathbf{1} \mathrm{e}^{-\beta\left(V_{01}+V_{11}\right)}\right]^{s} .
$$

The identity $\lim _{s \rightarrow 0} \partial x^{s} / \partial s=\lim _{s \rightarrow 0} \partial e^{s \ln x} / \partial s=\ln x$ (with $x$ being a dummy variable) is then used to express the QA free energy defined in Eq. (4) as

$$
-\beta F_{1}=\lim _{s \rightarrow 0} Z^{-1} \frac{\partial Z}{\partial s}=\lim _{s \rightarrow 0} \frac{\partial \ln Z}{\partial s},
$$

implying analytic continuation in $s$ (i.e., treating $s$ as a continuous parameter). The benefit of the replica trick is that Eq. (7) has the standard form of a partition sum, which avoids having to deal with the nested logarithm in Eq. (4). Of course, the limit (8) is still to be taken explicitly.

A corresponding procedure can be carried out, following Ref. 19, for the QA density functional. The Helmholtz free energy functional of the (equilibrium) replicated system is

$$
\mathcal{F}\left[\rho_{0}, \ldots, \rho_{s}\right]=\mathcal{F}_{\text {exc }}\left[\rho_{0}, \ldots, \rho_{s}\right]+\sum_{i=0}^{s} \mathcal{F}_{\text {id }}\left[\rho_{i}\right],
$$

where the free energy of an ideal gas of rotators (of species $i$ ) is

$$
\beta \mathcal{F}_{\mathrm{id}}\left[\rho_{i}\right]=\int d \mathbf{r} \int d \mathbf{u} \rho_{i}(\mathbf{r}, \mathbf{u})\left[\ln \left(\rho_{i}(\mathbf{r}, \mathbf{u}) \Lambda_{i}^{3}\right)-1\right]
$$

and the excess free energy functional up to second virial level, i.e., in the Onsager approximation, is

$$
\begin{aligned}
\beta \mathcal{F}_{\text {exc }}\left[\rho_{0}, \ldots, \rho_{s}\right]= & -\frac{1}{2} \rho_{0} \otimes f_{00} \otimes \rho_{0}-\sum_{i=1}^{s} \rho_{0} \otimes f_{0 i} \otimes \rho_{i} \\
& -\frac{1}{2} \sum_{i=1}^{s} \rho_{i} \otimes f_{i i} \otimes \rho_{i},
\end{aligned}
$$

where $f_{i j}=\exp \left(-\beta \phi_{i j}\right)-1$ is the Mayer bond (which equals -1 if the two particles involved overlap, and zero otherwise), and we have used a short-hand notation for the convolutions,

$$
\begin{aligned}
\rho_{i} \otimes f_{i j} \otimes \rho_{j} \equiv & \int d \mathbf{r} \int d \omega \int d \mathbf{r}^{\prime} \int d \omega^{\prime} \rho_{i}(\mathbf{r}, \mathbf{u}) \\
& \times f_{i j}\left(\mathbf{r}-\mathbf{r}^{\prime}, \mathbf{u}, \mathbf{u}^{\prime}\right) \rho_{j}\left(\mathbf{r}^{\prime}, \mathbf{u}^{\prime}\right) .
\end{aligned}
$$

Note that no terms that couple species $i>0$ and $j>0$, with $i \neq j$, appear in Eq. (11), as in these cases $\phi_{i j}=0$ and hence 
$f_{i j}=0$. If we assume the density profiles of all replicas to be the same, $\rho_{1}=\rho_{i}$ for $i>1$, then Eq. (11) reduces to

$$
\begin{aligned}
\beta \mathcal{F}_{\text {exc }}\left[\rho_{0}, \rho_{1}\right]= & -\frac{1}{2} \rho_{0} \otimes f_{00} \otimes \rho_{0}-s \rho_{0} \otimes f_{01} \otimes \rho_{1} \\
& -\frac{s}{2} \rho_{1} \otimes f_{11} \otimes \rho_{1} .
\end{aligned}
$$

Applying the limit $\mathcal{F}_{1}=\lim _{s \rightarrow 0} \partial \mathcal{F} / \partial s$ we obtain the QA free energy functional

$$
\beta \mathcal{F}_{1}\left[\rho_{0}, \rho_{1}\right]=\beta \mathcal{F}_{\mathrm{id}}\left[\rho_{0}\right]-\rho_{0} \otimes f_{01} \otimes \rho_{1}-\frac{1}{2} \rho_{1} \otimes f_{11} \otimes \rho_{1},
$$

where the second term describes the coupling of the annealed rods to the quenched rods and the third term is the contribution from interactions between pairs of annealed rods. The corresponding grand potential is

$$
\begin{aligned}
\Omega_{1}\left[\rho_{0}, \rho_{1}\right]= & \mathcal{F}_{1}\left[\rho_{0}, \rho_{1}\right]-\int d \mathbf{r} \int d \mathbf{u} \rho_{1}(\mathbf{r}, \mathbf{u})\left[V_{1}^{\mathrm{ext}}(\mathbf{r}, \mathbf{u})\right. \\
& \left.-\mu_{1}\right]
\end{aligned}
$$

where $V_{1}^{\text {ext }}(\mathbf{r}, \mathbf{u})$ is an external potential acting on positions and orientations of particles of species 1 . The minimization condition

$$
\left.\frac{\delta \Omega_{1}}{\delta \rho_{1}(\mathbf{r}, \mathbf{u})}\right|_{\rho_{0}\left(\mathbf{r}^{\prime}, \mathbf{u}^{\prime}\right)}=0
$$

determines $\rho_{1}(\mathbf{r}, \mathbf{u})$, when $\rho_{0}\left(\mathbf{r}^{\prime}, \mathbf{u}^{\prime}\right)$ is prescribed as a fixed density field (this can be obtained from an equilibrium DFT for the pure system of species 0 ). The second virial structure of Eq. (14) implies that the contribution of $\rho_{0}$ to the free energy is identical to the action of an (fictitious) external potential $V_{1}^{\mathrm{fic}}(\mathbf{r}, \mathbf{u}) \equiv \rho_{0} \otimes f_{01}$ that acts on species 1 . In an alternative model, where species 0 is taken to be an ideal gas of rotators with $V_{00}=0$, the first term in Eq. (13) vanishes, but this does not alter result (14) for the QA excess free energy. Note that in both models inhomogeneous density distributions of matrix rods (both in their positions and orientations) are allowed, as those can be generated from the action of an external field acting on species 0 before the quench.

\section{COMPUTATIONAL METHODS}

The numerical solution of the framework described above follows that used in previous work. ${ }^{38-40}$ In order to minimize the grand potential, i.e., solve Eq. (16), and hence determine the density profile, the orientationally dependent functions are expanded in spherical harmonics,

$$
\begin{aligned}
& \log \rho_{i}(\mathbf{r}, \mathbf{u})=\sum_{\ell, m} \tilde{\rho}_{\ell m}^{i}(\mathbf{r}) Y_{\ell m}(\mathbf{u}) \\
& \rho_{i}(\mathbf{r}, \mathbf{u})=\sum_{\ell, m} \rho_{\ell m}^{i}(\mathbf{r}) Y_{\ell m}^{*}(\mathbf{u})
\end{aligned}
$$

$$
V_{i}^{\mathrm{ext}}(\mathbf{r}, \mathbf{u})=\sum_{\ell, m} V_{\ell m}^{\mathrm{ext}, i}(\mathbf{r}) Y_{\ell m}(\mathbf{u})
$$

where again $i=0$ and 1 refer to quenched and annealed components, respectively, $Y_{\ell m}(\mathbf{u})$ is the spherical harmonic with indices $\ell m$, the asterisk in the expansion of density (17b) denotes the complex conjugate. The expansion coefficients of the respective quantities on the left hand side are $\tilde{\rho}_{\ell m}^{i}(\mathbf{r}), \rho_{\ell m}^{i}(\mathbf{r})$ and $V_{\ell m}^{\text {ext }, i}(\mathbf{r})$, and we restrict ourselves to $V_{i}^{\text {ext }}(\mathbf{r}, \mathbf{u})=0$ in the following. The Mayer function is expanded similarly in rotational invariants ${ }^{41}$

$$
f_{i j}\left(\Delta \mathbf{r}, \mathbf{u}, \mathbf{u}^{\prime}\right)=\sum_{\ell_{1}, \ell_{2}, \ell} f_{i j}^{\ell_{1} \ell_{2} \ell}(r) \Phi_{\ell_{1} \ell_{2} \ell}\left(\hat{\mathbf{r}}, \mathbf{u}, \mathbf{u}^{\prime}\right),
$$

where $\Delta \mathbf{r}$ denotes the difference vector between particle centers and $r=|\Delta \mathbf{r}|$ and $\hat{\mathbf{r}}=\Delta \mathbf{r} / r$. Note that for a bulk system the $\rho_{i}(\mathbf{r}, \mathbf{u})$, etc., are independent of position, i.e., $\rho_{i}(\mathbf{r}, \mathbf{u})$ $=\rho_{i}(\mathbf{u})$. Inserting the spherical harmonics expansions into the grand potential (15) and integrating over space gives

$$
\begin{aligned}
& \frac{\beta \Omega_{1}\left[\rho_{0}(\mathbf{r}, \mathbf{u}), \rho_{1}(\mathbf{r}, \mathbf{u})\right]}{V} \\
& =\sum_{\ell, m} \rho_{\ell m}^{1}\left\{\widetilde{\rho}_{\ell m}^{1}-\sqrt{4 \pi}\left(1+\beta \mu_{1}\right) \delta_{\ell 0}+V_{\ell m}^{1}\right\} \\
& \quad+\sum_{\ell_{1}, \ell_{2}, m}\left\{V_{\ell_{1} \ell_{2} m}^{11} \rho_{\ell_{1} m}^{1} \rho_{\ell_{2} m}^{1}+2 V_{\ell_{1} \ell_{2} m}^{10} \rho_{\ell_{1} m}^{1} \rho_{\ell_{2} m}^{0}\right\},
\end{aligned}
$$

where $V$ is the total system volume and $V_{\ell_{1} \ell_{2} m}^{i j}$ are orientationally averaged components of the excluded volume between species $i$ and $j$ defined as $V_{\ell_{1} \ell_{2} m}^{i j}$ $=-(-1)^{m} 4 \pi \int d r r^{2} f_{i j}^{\ell_{1} \ell_{2} 0}(r)$; note that $f_{i j}^{\ell_{1} \ell_{2} m}=(-1)^{m} f_{i j}^{\ell_{1} \ell_{2} 0}$. For the case of spatial dependence on a single Cartesian variable $z$, inserting the expansions into the grand potential gives

$$
\begin{aligned}
& \frac{\beta \Omega_{1}\left[\rho_{0}(\mathbf{r}, \mathbf{u}), \rho_{1}(\mathbf{r}, \mathbf{u})\right]}{A} \\
& =\int d z \rho_{\ell m}^{1}(z)\left\{\tilde{\boldsymbol{\rho}}_{\ell m}^{1}(z)+V_{\ell m}^{i}(z)-\sqrt{4 \pi}\left(1+\beta \mu_{1}\right) \delta_{\ell 0}\right\} \\
& \quad+\int d z_{1} d z_{2} \sum_{\ell_{1} \ell_{2} m}\left\{A_{\ell_{1} \ell_{2} m}^{11}\left(z_{12}\right) \rho_{\ell_{1} m}^{1}\left(z_{1}\right) \rho_{\ell_{2} m}^{1}\left(z_{2}\right)\right. \\
& \left.\quad+2 A_{\ell_{1} \ell_{2} m}^{10}\left(z_{12}\right) \rho_{\ell_{1} m}^{1}\left(z_{1}\right) \rho_{\ell_{2} m}^{0}\left(z_{2}\right)\right\},
\end{aligned}
$$

where $A$ is the lateral system size (normal to the $z$-direction), and $z_{12}=z_{1}-z_{2}$ and $A_{\ell_{1} \ell_{2} m}^{i j}(z)$ are components of the pair excluded area for molecules of separation $z$. As the second term (excess free energy) in Eq. (20) is a convolution, it is most conveniently evaluated in reciprocal space. ${ }^{42}$

The equilibrium density is found by numerically minimizing the grand potential, using the conjugate gradients method, ${ }^{43}$ with respect to $\tilde{\rho}_{\ell m}(z)$. When required, the $\rho_{\ell m}(z)$ coefficients are determined from Eq. (17), with angular integration performed using Lebedev quadrature ${ }^{44}$ with 302 angular grid points. For numerical calculations the integrals are replaced by sums over a regular grid. The calculations are performed on a grid of 1500 points, with a spacing of $\Delta z$ $=0.2 \mathrm{~b}$ and periodic boundary conditions. The spherical harmonics expansions are truncated at $\ell_{\max }=6$ for $\log \rho(z, \mathbf{u})$ 
and $\ell_{\max }=8$ for $\rho(z, \mathbf{u})$. For studying the interfacial properties the quenched hard rods are confined in a region of width $100 b=6.67 a$, with aspect ratio $a / b=15$. Good agreement is found between the Onsager theory and simulations for this aspect ratio (see, e.g., Ref. 45) and while typical liquid crystal molecules have somewhat shorter aspect ratios $(e \approx 4-8)$, the behavior of such shorter molecules is expected to be qualitatively similar.

\section{RESULTS}

In the case of uniform and isotropic matrices of quenched rods, i.e., $\rho_{0}=$ const, the free energy functional (14) reduces to that of the pure system of species 1 , but with an additional additive term that is linear in $\rho_{1}$. As a consequence, the isotropic-nematic transition persists with unchanged isotropic and nematic coexistence densities $\rho_{1}^{\text {iso }}$ and $\rho_{1}^{\text {nem }}$, and only the chemical potential at the transition is shifted by a constant $\Delta \mu=k_{B} T V_{000}^{01} \rho_{0}$, where $V_{000}^{01}$ $=298.087 b^{3}=1.32483 a^{2} b$ is the orientationally averaged excluded volume of two ellipsoids of species 0 and 1 .

We investigate interfacial behavior of the annealed hard rods at a surface of the porous matrix, modeled as a step function distribution of the centers of the quenched rods with isotropic orientations, $\rho_{0}(\mathbf{r}, \mathbf{u})=\rho_{0} \Theta(z)$, where $\Theta(\cdot)$ is the Heaviside function and $\rho_{0}$ is the density of quenched rods that constitute the matrix which is located at $z>0$. We consider equal-sized quenched and annealed rods, $a_{0}=a_{1} \equiv a$ and $b_{0}=b_{1} \equiv b$, and have chosen the aspect ratio to be $a / b=15$. For computational reasons, the system is periodic in the $z$-direction, with period $20 a$ such that $-13.33 a<z<6.67 a$ (and in units of rod thickness $-200 b<z<100 b$ ). The matrix fills the region $0<z<6.67 b$. From the full density distribution of species 1 we obtain the orientation-averaged density profile of the particle centers, $\rho(z)=\int d \underline{\mathbf{u}} \rho_{1}(z, \mathbf{u})$, which we normalize $\rho^{*}(z)=\rho(z) / \rho_{\text {cp }}$, where $\rho_{\text {cp }}=\sqrt{2} /\left(a b^{2}\right)$ is the closepacked density. The nematic order parameter profile is $S(z)$ $=\rho(z)^{-1} \int d \mathbf{u} \rho_{1}(z, \mathbf{u}) P_{2}(\mathbf{u} \cdot \mathbf{n})$, where $\mathbf{n}$ is the nematic director and $P_{2}(x)=\left(3 x^{2}-1\right) / 2$ is the second Legendre polynomial. Both the order parameter profile and the biaxiality profile $S_{x y}(z)$, which is obtained from the nematic order tensor,

$$
Q_{\alpha \beta}(z)=\frac{3}{2} \int d \mathbf{u} \rho_{1}(z, \mathbf{u}) u_{\alpha} u_{\beta}-\frac{1}{2} \delta_{\alpha \beta},
$$

where $\delta_{\alpha \beta}$ is the Kronecker delta and the Greek indices enumerate the Cartesian directions. The order parameters are found from diagonalizing $Q_{\alpha \beta}(z)$ and sorting the (three) eigenvalues in magnitude: The largest eigenvalue is identified with $S(z)$, the difference between the middle and the smallest one constitutes $S_{x y}(z)$. We show results for two representative values of the matrix density: $\rho_{0} b^{3}=0.001$ and $\rho_{0} b^{3}=0.1$. We first consider the case of normal alignment of the nematic director far from the surface and the surface normal, $\mathbf{n}=\hat{\mathbf{z}}$, where $\hat{\mathbf{z}}$ is the unit vector in the $z$-direction. Figure 1 displays results for $\rho^{*}(z)$ and $S(z)$ for the case of very low matrix density, $\rho_{0} b^{3}=0.001$, and a range of increasing values of the chemical potential for the annealed species $1, \beta \mu \equiv \beta \mu_{1}$ $=1.2-1.4$. For values of $\mu$ below the value at isotropicnematic bulk coexistence of the pure system (without ma-
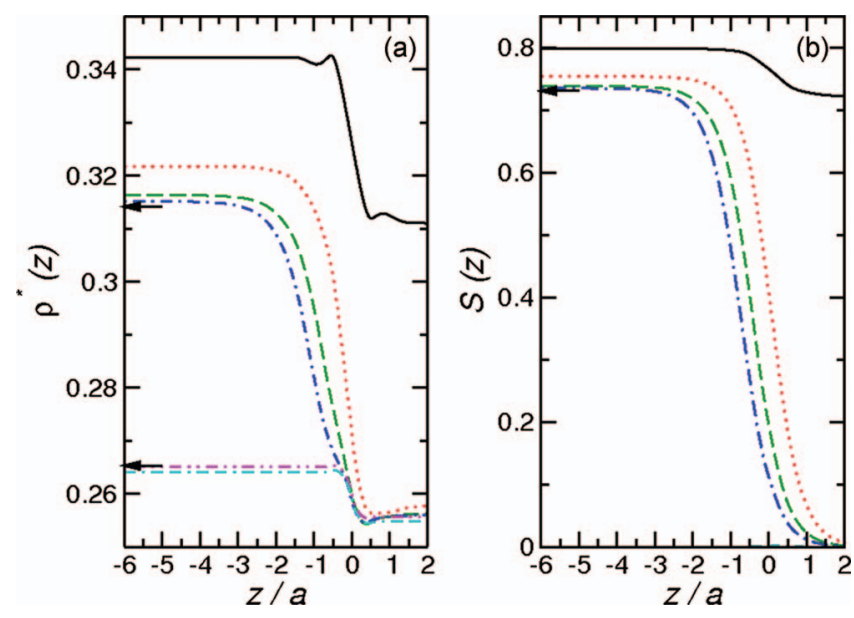

FIG. 1. (a) Scaled density profile $\rho^{*}(z)=\rho(z) / \rho_{\text {cp }}$ and (b) order parameter profile $S(z)$ of annealed hard rods at the surface of a quenched hard rod matrix of density $\rho_{0} b^{3}=0.001$ (for elongation $e=a / b=15$ ), with homeotropic alignment far from the surface. Results for chemical potentials (for annealed rods) $\beta \mu_{1}=1.50$ (solid black line), 1.30 (dotted red line), 1.25 (dashed green line), 1.24 (dotted-dashed blue line), 1.23 (double-dotted-dashed magenta line), and 1.20 (dotted-double-dashed cyan line). The coordinate $z$ measures distance to the matrix surface and arrows denote coexistence values for $\rho^{*}$ and $S$.

trix), $\rho(z)$ crosses over smoothly from outside $(z<0)$ to smaller values inside $(z>0)$ the matrix, see the results for the density profile of particle centers in Fig. 1(a). The observed reduction in density for $z>0$ can clearly be attributed to the repulsion that the quenched rods exert on species 1 . Increasing $\mu$ to values above the isotropic-nematic transition induces a jump in the density outside. However, as the rods inside the matrix are still in an isotropic state, their density is hardly affected. The corresponding order parameter profile, shown in Fig. 1(b), displays smooth crossover from values close to the nematic coexistence value to zero inside the matrix. Increasing the chemical potential further to $\beta \mu$ $=1.50$ leads to nematization inside the matrix. Hence the density inside the matrix increases considerably, as compared to the case above, and the order parameter becomes finite. The density profile shows oscillations both inside and outside the matrix. The value of the chemical potential is
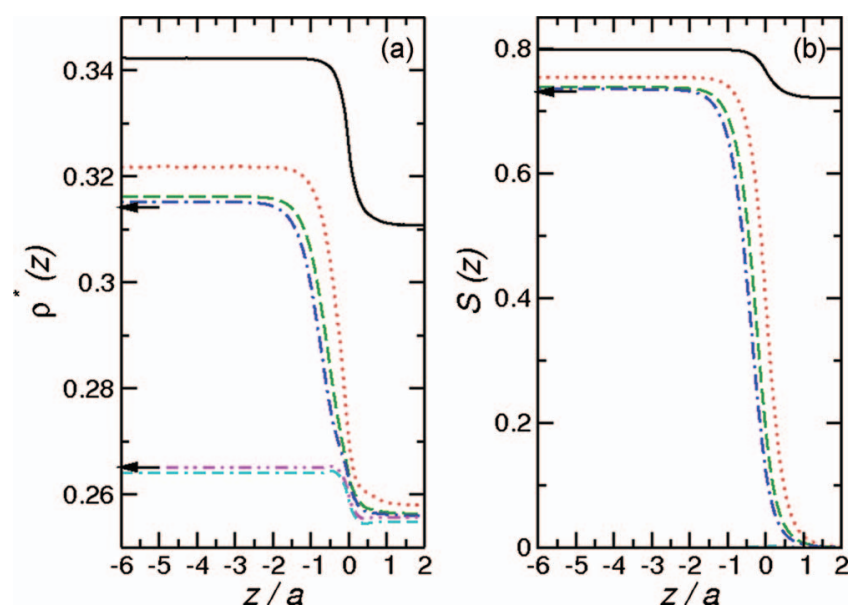

FIG. 2. Same as Fig. 1, but for planar alignment of the nematic director far from the matrix. 
slightly lower than the coexistence chemical potential of hard rods in an infinite bulk matrix of this density, which is at $\beta \mu=1.53$. We attribute this difference to the finite width of the porous matrix. The effect can be quantified on the basis of the Kelvin equation for capillary nematization,

$$
\Delta \mu_{\mathrm{coex}}=\frac{2 \gamma_{I N}}{\left(\rho_{N}-\rho_{I}\right) L},
$$

where $\Delta \mu_{\text {coex }}$ is the shift of the coexistence chemical potential of the confined with respect to the infinite system, $\gamma_{I N}$ is the interface tension of the free interface between isotropic and nematic phases, $\rho_{N} b^{3}=0.029645$ and $\rho_{I} b^{3}=0.025008$ are the coexistence densities in the nematic and isotropic phase, respectively ${ }^{45}$ (hence $\rho_{N} b^{3}-\rho_{I} b^{3}=0.004$ 637), and $L=6.67 a$ is the width of the matrix. For homeotropic alignment of the director with the (free) IN interface $\gamma_{I N}=0.016 k_{B} T / b^{2} \quad$ (and for planar alignment $\left.\gamma_{I N}=0.010 k_{B} T / b^{2}\right)^{45}$ Hence the shift is $\beta \Delta \mu_{\text {coex }}=0.07$ (for planar alignment 0.043), which compares reasonably well with results from directly calculating the grand potential difference between systems with nematic or isotropic ordering inside the matrix; this gives a transition at $\beta \mu_{\text {coex }}=1.44$ (1.48) for homeotropic (planar) alignment. Note that taking the value of the free $I N$ interface in Eq. (22) constitutes a further approximation, as in the actual physical situation the $I N$ interface is "pinned" at the porous surface of the matrix. Although the value of the chemical potential at coexistence changes, within a second virial treatment this is not the case for the coexistence densities.

We next consider the boundary conditions at $z \rightarrow-\infty$ such that the nematic director possesses planar alignment with respect to the matrix surface, hence $\mathbf{n} \perp \hat{\mathbf{z}}$, and investigate the resulting behavior for the same sequence of chemical potentials of species 1 as above. In this situation the boundary values of $\rho(z)$ and $S(z)$ at $z \rightarrow \pm \infty$ are the same as before. While the interfacial behavior is qualitatively similar, the crossover region possesses a smaller interfacial width. Moreover, both $\rho(z)$ and $S(z)$ decay monotonically for the highest value of the chemical potential considered, where the entire system is in a nematic state.

Increasing the matrix density to $\rho_{0} b^{3}=0.1$ has the pronounced effect that the adsorption inside the matrix becomes very small, and the matrix essentially acts like a rough, hard wall on the fluid, see Fig. 3 for results where the alignment far from the matrix is homeotropic, $\mathbf{n}=\hat{\mathbf{z}}$. We find a thin layer of rods aligned parallel to the matrix surface, indicated by a peak in the density and order parameter profiles at the matrix surface. This is consistent with the alignment of hard ellipsoids at the free isotropic-nematic interface in equilibrium. ${ }^{35,46}$ In the case of parallel alignment, see Fig. 4, the decrease in $\rho(z)$ and $S(z)$, as observed in the case above, is weaker and occurs much closer to the matrix surface. In both cases, there is virtually no adsorption inside the matrix.

We show results for the biaxiality profile $S_{x y}(z)$ in Fig. 5. These indicate that there is small biaxiality at high matrix densities, while $S_{x y}$ is essentially zero at low densities this. A peak at the surface is apparent. When the fluid outside is isotropic a small shoulder is apparent. For homeotropic
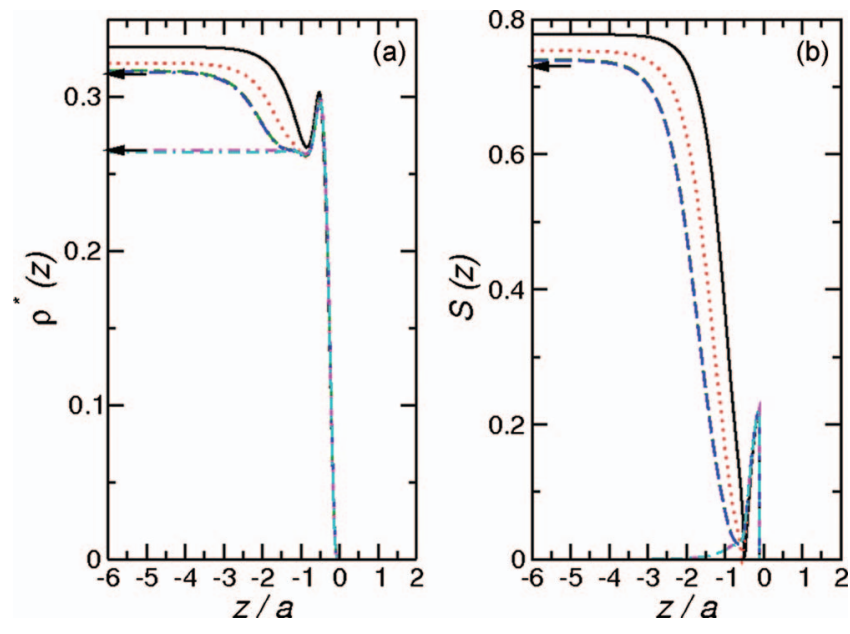

FIG. 3. Same as Fig. 1, but for matrix density $\rho_{0} b^{3}=0.10$.

alignment the width of this peak grows as the chemical potential grows toward its value at coexistence.

The behavior of $S_{x y}(z)$, in particular, its sign, has often been used to infer details of the orientational behavior of liquid crystals at interfaces and surfaces. ${ }^{35}$ From the definition of the biaxiality used here, $S_{x y}(z)$ is strictly positive; however, similar information may be gained from the eigenvectors corresponding to the second and third eigenvalues (middle and smallest respectively) of the order tensor. For planar alignment these eigenvectors interchange their identity at the interface between the bulk nematic and surface isotropic regions, cf. Fig. 5. On the bulk side the second eigenvector lies along the $z$ axis, indicating that the rods stick through the interface, while on the isotropic side the second eigenvector lies along the $y$ axis. For homeotropic alignment the second eigenvector is along the $y$ axis on the nematic side and along the $x$ axis on the isotropic side (due to the cylindrical symmetry around the $z$ axis these two states are equivalent). The biaxiality profiles are strictly positive due to this effect. In contrast, using space-fixed axes (i.e., not allowing the second and third eigenvectors to interchange their identities) leads a small region of negative biaxiality (near $z \approx 0.5 a$ ) for the case of planar alignment. We conclude that
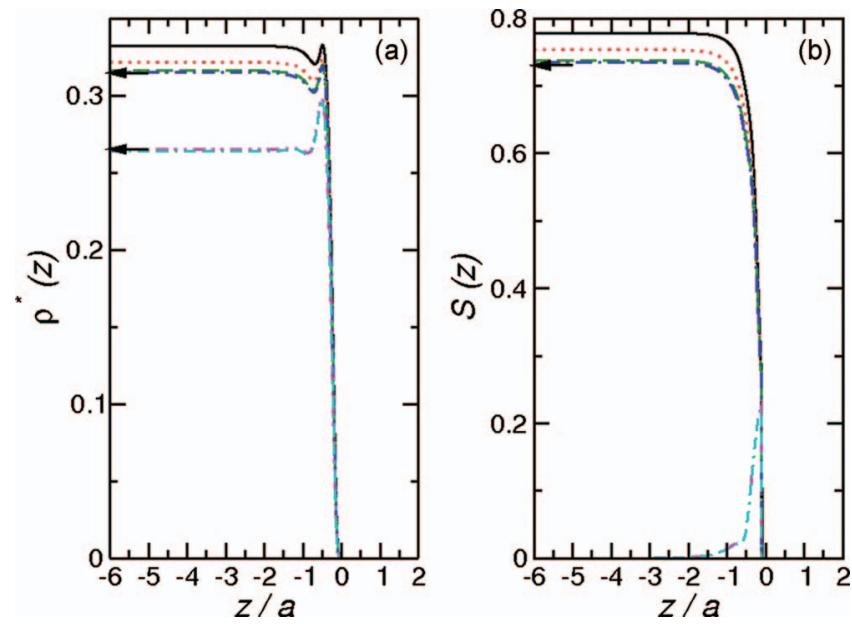

FIG. 4. Same as Fig. 2 (planar alignment of the nematic director far from the matrix), but for matrix density $\rho_{0} b^{3}=0.1$ (as in Fig. 3). 

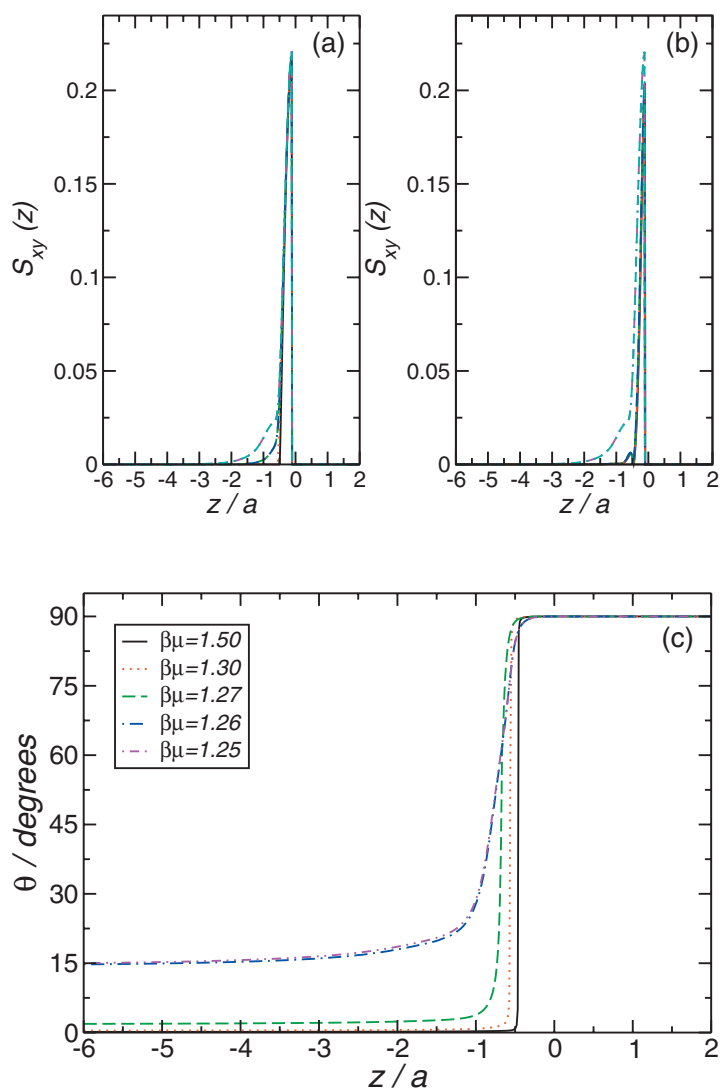

FIG. 5. Biaxiality profiles, $S_{x y}(z)$, for annealed hard rods with (a) homeotropic and (b) planar alignment far from the matrix surface. Results for $\beta \mu_{1}=1.50$ (solid black line), 1.30 (red dotted line), 1.25 (green dashed line), 1.24 (blue dotted-dashed line), 1.23 (magenta double-dotted-dashed line), and 1.20 (cyan dotted-double-dashed line). Also shown is the tilt angle $\theta(\mathrm{c})$ between the nematic director and the $z$ axis for the case of homeotropic alignment.

the biaxial properties are similar to previous simulation and theoretical results for the free nematic-isotropic interface, with the region of negative biaxiality (without exchange of eigenvector identity) lying on the nematic side of the interface, which indicates that there is a small preference for molecules to stick through the interface. ${ }^{35,46}$ The peak in $S_{x y}(z)$ on the isotropic size is significantly larger than what is found at the free interface due to the influence of the matrix surface.

Finally, we display in Fig. 6 the density and order parameter profiles for fixed value of the chemical potential of the annealed rods, $\beta \mu=1.60$, and varying matrix density $\rho_{0}$. Upon increasing $\rho_{0}$ the density inside the matrix decreases and the variation with $z$ in the interfacial region becomes more rapid. This indicates that the fluid is being expelled from the matrix upon increasing $\rho_{0}$. Different cases are shown, ranging from very low matrix density with nematic fluid inside $\left(\rho b^{3}=0.001\right)$, to slightly denser matrices which have isotropic fluid inside $\left(\rho b^{3}=0.002,0.01\right)$, and denser matrices with very low or essentially zero density inside.

\section{CONCLUSIONS}

In conclusion we have investigated bulk and interfacial isotropic-nematic behavior for a simple hard core model of mobile rods that are immersed inside and at the boundary of
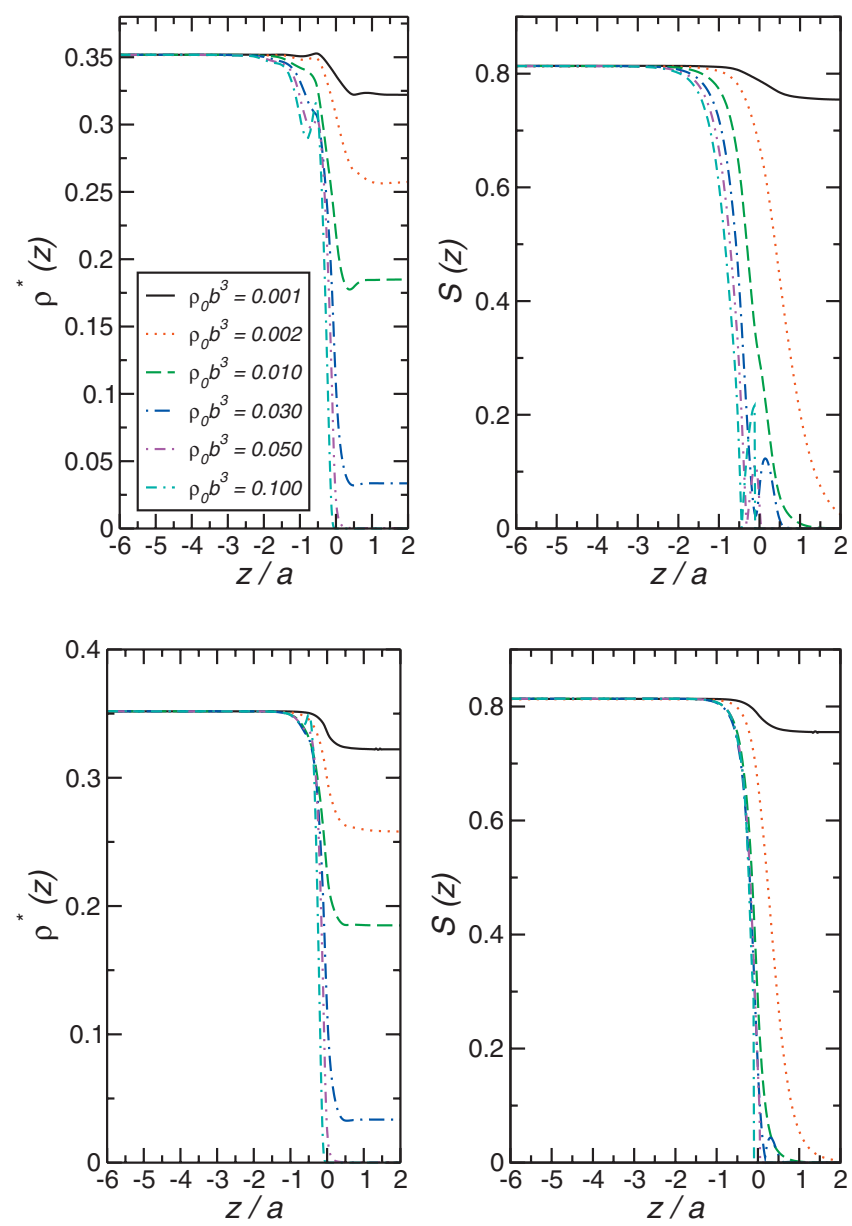

FIG. 6. Density profile and nematic order parameter profile for fixed chemical potential $\beta \mu=1.60$ and increasing values of the density of the quenched rods, $\rho_{0}$ (as indicated), for homeotropic (top) and planar (bottom) alignments far from the matrix.

a matrix that is formed by rods with quenched positions and orientations. This model could be taken to represent adsorption of dispersed colloidal rods in a matrix of entangled colloidal rods or inside a fibrillar porous substance. Our results, based on a simple second virial approximation to the QA excess free energy functional, indicate rich microscopic structure close to the surface of the matrix. Within this meanfield-like treatment the isotropic-nematic transition persists inside the matrix but is shifted toward higher values of the chemical potential. It would be very interesting to test these findings using computer simulations. Furthermore one could envisage experimental realizations, where colloidal rods form an entangled matrix, which is penetrated by a lyotropic liquid crystal, similar to setups where self-diffusion and sedimentation of tracer spheres in semidilute dispersions of rigid colloidal rods ${ }^{47}$ were investigated. In the current work we restricted ourselves to studying isotropic matrices. A different type of influence on the annealed rods would be exerted by anisotropic matrices of rods, e.g., such that the matrix itself possesses (quenched) nematic order. Based on the structure of the second virial excess free energy functional and the fact that within this approach the matrix acts as an effective external field, one could expect an aligning effect on the mobile rods. This should have interesting conse- 
quences for the bulk phase diagram. We leave this problem to possible future work.

\section{ACKNOWLEDGMENTS}

This work was supported by the UK EPSRC and the Liquid Crystals Group of HP Laboratories, Bristol through a CASE award. Computational resources for this work were provided by the Centre for Scientific Computing, University of Warwick. We thank S. Klein and C. J. P. Newton for useful discussions.

${ }^{1}$ T. Bellini, M. Buscaglia, C. Chiccoli, F. Mantegazza, P. Pasini, and C. Zannoni, Phys. Rev. Lett. 85, 1008 (2000).

${ }^{2}$ T. Bellini, M. Buscaglia, C. Chiccoli, F. Mantegazza, P. Pasini, and C. Zannoni, Phys. Rev. Lett. 88, 245506 (2002).

${ }^{3}$ Y. Imry and S. Ma, Phys. Rev. Lett. 35, 1399 (1975).

${ }^{4}$ D. E. Feldman, Phys. Rev. Lett. 84, 4886 (2000).

${ }^{5}$ L. Onsager, Ann. N. Y. Acad. Sci. 51, 627 (1949).

${ }^{6}$ G. J. Vroege and H. N. W. Lekkerkerker, Rep. Prog. Phys. 55, 1241 (1992).

${ }^{7}$ M. Franco-Melgar, A. J. Haslam, and G. Jackson, Mol. Phys. 106, 649 (2008).

${ }^{8}$ R. Evans, Adv. Phys. 28, 143 (1979).

${ }^{9}$ R. Evans, in Fundamentals of Inhomogeneous Fluids, edited by D. Henderson (Dekker, New York, 1992), Chap. 3, p. 85.

${ }^{10}$ W. G. Madden and E. D. Glandt, J. Stat. Phys. 51, 537 (1988).

${ }^{11}$ J. A. Given and G. Stell, J. Chem. Phys. 97, 4573 (1992).

${ }^{12}$ M. L. Rosinberg, G. Tarjus, and G. Stell, J. Chem. Phys. 100, 5172 (1994).

${ }^{13}$ C. Spöler and S. H. L. Klapp, J. Chem. Phys. 118, 3628 (2003).

${ }^{14}$ C. Spöler and S. H. L. Klapp, J. Chem. Phys. 120, 6734 (2004).

${ }^{15}$ C. Spöler and S. H. L. Klapp, J. Chem. Phys. 121, 9623 (2004).

${ }^{16}$ G. I. Menon and C. Dasgupta, Phys. Rev. Lett. 73, 1023 (1994).

${ }^{17}$ F. Thalmann, C. Dasgupta, and D. Feinberg, Europhys. Lett. 50, 54 (2000).

${ }^{18}$ M. Schmidt, Phys. Rev. E 66, 041108 (2002).

${ }^{19}$ H. Reich and M. Schmidt, J. Stat. Phys. 116, 1683 (2004).
${ }^{20}$ L. Lafuente and J. A. Cuesta, Phys. Rev. E 74, 041502 (2006).

${ }^{21}$ P. I. Ravikovitch and A. V. Neimark, Langmuir 22, 11171 (2006).

${ }^{22}$ A. J. Archer, M. Schmidt, and R. Evans, Phys. Rev. E 73, 011506 (2006).

${ }^{23}$ A. V. Neimark, Y. Lin, P. I. Ravikovitch, and M. Thommes, Carbon 47, 1617 (2009)

${ }^{24}$ M. Schmidt, J. Phys.: Condens. Matter 17, S3481 (2005).

${ }^{25}$ P. Bryk, W. Rzysko, A. Malijevsky, and S. Sokołowski, J. Colloid Interface Sci. 313, 41 (2007).

${ }^{26}$ M. Matusewicz, A. Patrykiejew, S. Sokołowski, and O. Pizio, J. Chem. Phys. 127, 174707 (2007).

${ }^{27}$ Z. Sokołowska and S. Solołowski, J. Colloid Interface Sci. 316, 652 (2007).

${ }^{28}$ A. Patrykiejew, S. Sokołowski, R. Tscheliessnig, and J. Fischer, J. Phys. Chem. B 112, 4552 (2008).

${ }^{29}$ M. Schmidt and J. M. Brader, J. Chem. Phys. 119, 3495 (2003).

${ }^{30}$ M. Schmidt and M. Dijkstra, J. Chem. Phys. 121, 12067 (2004).

${ }^{31}$ D. L. Cheung and F. Schmid, J. Chem. Phys. 122, 074902 (2005).

${ }^{32}$ D. L. Cheung and F. Schmid, Chem. Phys. Lett. 418, 392 (2006).

${ }^{33}$ R. van Roij and B. Mulder, Phys. Rev. E 54, 6430 (1996).

${ }^{34}$ R. van Roij and B. Mulder, J. Chem. Phys. 105, 11237 (1996).

${ }^{35}$ K. Shundyak and R. van Roij, J. Phys.: Condens. Matter 13, 4789 (2001).

${ }^{36}$ K. Shundyak and R. van Roij, Phys. Rev. Lett. 88, 205501 (2002).

${ }^{37}$ K. Shundyak and R. van Roij, Phys. Rev. E 68, 061703 (2003).

${ }^{38}$ M. P. Allen, Mol. Phys. 96, 1391 (1999).

${ }^{39}$ D. Andrienko and M. P. Allen, Phys. Rev. E 65, 021704 (2002).

${ }^{40}$ D. L. Cheung and F. Schmid, J. Chem. Phys. 120, 9185 (2004).

${ }^{41}$ C. G. Gray and K. E. Gubbins, Theory of Molecular Fluids. 1. Fundamentals (Clarendon, Oxford, 1984).

${ }^{42}$ D. L. Cheung and M. P. Allen, Phys. Rev. E 74, 021701 (2006).

${ }^{43}$ W. H. Press, B. P. Flannery, S. A. Teukolsky, and W. T. Vetterling, Numerical Recipes in Fortran, 2nd ed. (Cambridge University Press, Cambridge, 1992)

${ }^{44}$ V. I. Lebedev, Sib. Math. J. 18, 99 (1977).

${ }^{45}$ A. J. McDonald, M. P. Allen, and F. Schmid, Phys. Rev. E 63, 010701 (2000).

${ }^{46}$ N. Akino, F. Schmid, and M. P. Allen, Phys. Rev. E 63, 041706 (2001).

${ }^{47}$ S. G. J. M. Kluijtmans, G. H. Koenderink, and A. P. Philipse, Phys. Rev. E 61, 626 (2000) 